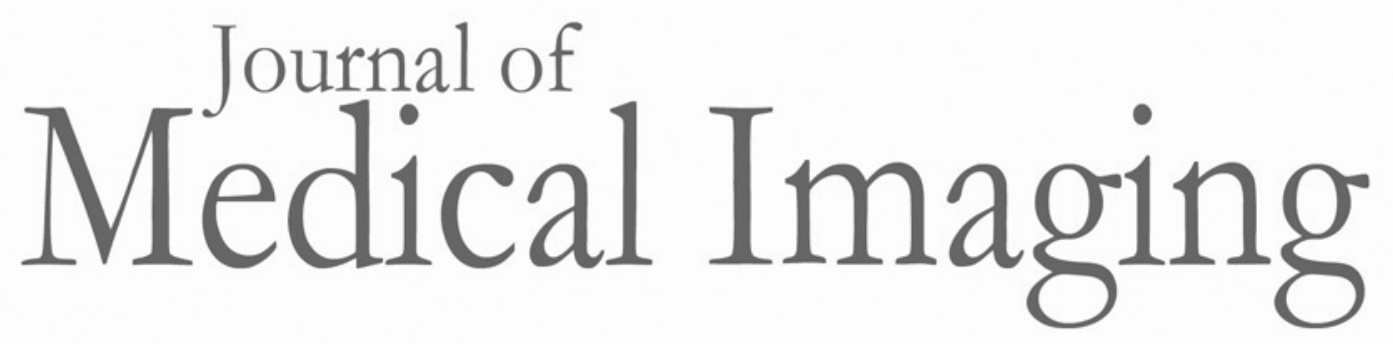

Medicallmaging.SPIEDigitalLibrary.org

\title{
Cancer cell classification with coherent diffraction imaging using an extreme ultraviolet radiation source
}

Michael Zürch

Stefan Foertsch

Mark Matzas

Katharina Pachmann

Rainer Kuth

Christian Spielmann

\section{SPIE.}




\title{
Cancer cell classification with coherent diffraction imaging using an extreme ultraviolet radiation source
}

\author{
Michael Zürch, ${ }^{\mathrm{a}, \star}$ Stefan Foertsch, ${ }^{\mathrm{b}}$ Mark Matzas, ${ }^{\mathrm{c}}$ Katharina Pachmann, ${ }^{\mathrm{d}}$ Rainer Kuth, ${ }^{\mathrm{b}}$ and \\ Christian Spielmann ${ }^{\mathrm{a}}$ \\ ${ }^{a}$ Friedrich-Schiller-University Jena, Institute of Optics and Quantum Electronics, Abbe Center of Photonics, Max-Wien-Platz 1, \\ Jena 07743 , Germany \\ bSiemens AG, Healthcare Sector Strategy, Hartmann Street 16, Erlangen 91052, Germany \\ 'Siemens AG, Corporate Technology, Günther-Scharowsky-Street 1, Erlangen 91058, Germany \\ dUniversity Hospital Jena, Bachstrasse 18, Jena 07743, Germany
}

\begin{abstract}
In cancer treatment, it is highly desirable to classify single cancer cells in real time. The standard method is polymerase chain reaction requiring a substantial amount of resources and time. Here, we present an innovative approach for rapidly classifying different cell types: we measure the diffraction pattern of a single cell illuminated with coherent extreme ultraviolet (XUV) laser-generated radiation. These patterns allow distinguishing different breast cancer cell types in a subsequent step. Moreover, the morphology of the object can be retrieved from the diffraction pattern with submicron resolution. In a proof-of-principle experiment, we prepared single MCF7 and SKBR3 breast cancer cells on gold-coated silica slides. The output of a laser-driven XUV light source is focused onto a single unstained and unlabeled cancer cell. With the resulting diffraction pattern, we could clearly identify the different cell types. With an improved setup, it will not only be feasible to classify circulating tumor cells with a high throughput, but also to identify smaller objects such as bacteria or even viruses. () The Authors. Published by SPIE under a Creative Commons Attribution 3.0 Unported License. Distribution or reproduction of this work in whole or in part requires full attribution of the original publication, including its DOI. [DOI: 10.1117/1.JMI.1.3.031008]
\end{abstract}

Keywords: breast cancer; rapid classification; high harmonic generation; coherent diffraction imaging; high resolution imaging. Paper 14032SSPRR received Mar. 27, 2014; revised manuscript received Aug. 12, 2014; accepted for publication Sep. 15, 2014; published online Oct. 3, 2014.

\section{Introduction}

Streaming the DNA of a cell with polymerase chain reaction (PCR) analysis is currently the most reliable method to determine the expression profile of a cell. For instance, in the clinical treatment of cancer, this information is necessary to determine the chemical agent to be used in chemotherapy or to determine the risk of spread of malignant cells across the body. However, PCR analysis requires a substantial amount of resources and time. Typical round-trip times from submitting a tissue sample until report reception are several days. ${ }^{1}$ Therefore, a faster and possibly cheaper method for on-site classification will be highly appreciated.

Current research activities for developing a method for the fast discrimination of different cells focus on spectroscopic methods such as Raman spectroscopy. ${ }^{2}$ Here, we present a different approach which is based on coherent diffraction imaging (CDI). ${ }^{3,4}$ The field of CDI emerged with the advent of synchrotrons delivering coherent radiation at short wavelengths down into the $\mathrm{x}$-ray regime. Nowadays, microscopy with a resolution down to the scale of proteins $\mathrm{s}^{5,6}$ is feasible at large-scale facilities such as synchrotrons and more recently at x-ray free-electron lasers. ${ }^{7-9}$ However, for the routine operation in a clinical environment drawing on these large-scale facilities is not an option.

A very promising approach for coherent $\mathrm{X}$-ray imaging techniques on a lab scale is frequency upconversion of ultrafast laser light. A process called high harmonic generation $(\mathrm{HHG})^{10-12}$ is suitable to generate coherent XUV and soft $\mathrm{X}$-ray radiation down

*Address all correspondence to: Michael Zürch, E-mail: michael.zuerch@unijena.de to a wavelength of a few nanometers at a sufficient photon flux to image samples at a high resolution. In this paper, we present a tabletop CDI setup that operates in reflection geometry at $38-\mathrm{nm}$ wavelength. We employed the setup in a first proofof-principle experiment to take diffraction patterns of breast cancer cell line cells and present a method that allows the discrimination of different breast cancer cell expressions from their diffraction patterns. Additionally, we demonstrate the reconstruction of a high resolution image of the specimen from the diffraction pattern.

\section{Experimental Setup}

Our experimental setup ${ }^{13,14}$ consists of a commercial ultrafast infrared laser delivering light pulses with a pulse duration below $30 \mathrm{fs}$ and $700 \mu \mathrm{J}$ of energy, see Fig. 1(a). The output pulses are focused into an argon jet. The high intensity in the focus $\left(\sim 10^{14} \mathrm{~W} / \mathrm{cm}^{2}\right)$ is sufficient for nonlinear frequency conversion of the laser pulses via HHG. The output consists of a highly coherent laser-like light beam with a spectrum containing all odd harmonics of the driving laser frequency between 20 and $800 \mathrm{~nm}$ [Fig. 1(b)]. It is worth mentioning that the generated XUV light takes on the spatial coherence of the driving laser beam, i.e., the XUV radiation is emitted in a beam with a low divergence and a well-defined wavefront. A thin aluminum foil blocks the strong 800-nm light and is transparent for XUV light in the range of 20 to $80 \mathrm{~nm}$. The transmitted XUV light is refocused onto the sample with a gold coated toroidal mirror. A grating between the focusing mirror and the sample is used to select a single harmonic out of the spectrum. In the rear focal plane of the toroidal mirror, the different harmonic lines are not 
(a)

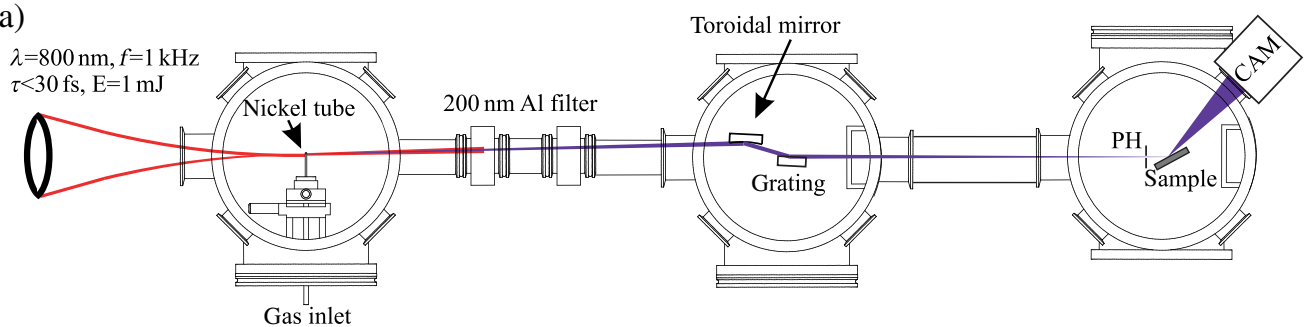

(b)

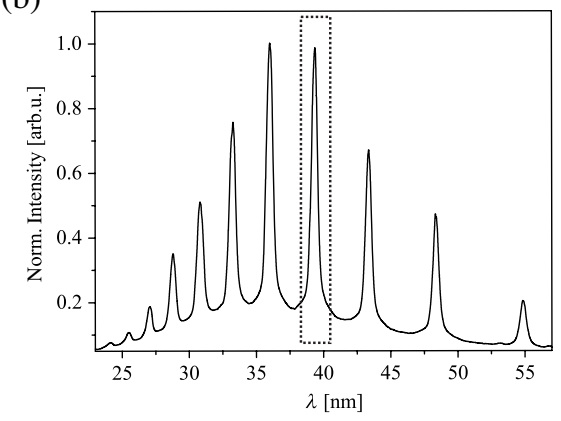

Fig. 1 The experimental setup and HHG spectrum. (a) The ultrafast infrared laser pulses are focused and the XUV radiation is generated in a nickel tube that is continuously fed with argon gas. Downstream a toroidal mirror refocuses the light. A grating disperses the contained wavelengths. A pinhole (PH) selects the monochromatic focus in the rear focal plane of the toroidal mirror. The specimen on a sample mount sits directly behind the pinhole. The diffracted light is measured with a CCD camera (CAM) in reflection geometry. The overall setup fits on an optical table 3 by $1-\mathrm{m}$ wide. (b) With this setup a single harmonic line can be selected out of the generated HHG spectrum (dotted rectangle).

only focused but are also spatially separated. With a $5-\mu \mathrm{m}$ pinhole (PH) just before the rear focal plane, we can select the wavelength of the radiation illuminating the sample. Additionally, the $\mathrm{PH}$ ensures a well-defined illuminating beam diameter with a slightly curved wave front. This arrangement for illumination and diffraction is known as Fresnel diffraction imaging and represents a mixed regime of holography and coherent diffraction imaging. ${ }^{15,16}$ This scheme is advantageous because it does not require a beam stop to suppress the strong central speckle, as it is necessary for $\mathrm{CDI},{ }^{17}$ and it allows capturing useful coherent diffraction data on the whole area of the detector.

The sample is positioned behind the $\mathrm{PH}$ and the coherently diffracted light is captured with an x-ray sensitive charge coupled device (CCD) camera $365 \mathrm{~mm}$ away from the sample. From the captured diffraction pattern one could reconstruct a real space image of the object, ${ }^{18,19}$ which, however, is expensive in computation time. However, recently we demonstrated that this setup is capable of recording well-resolved images of nanostructured samples in reflection geometry. ${ }^{13}$

Using reflection geometry for CDI at XUV wavelengths has several advantages. ${ }^{13,20,21}$ The geometry was chosen because there are no easy-to-handle rigid substrates that are also transparent for XUV radiation. Therefore, transmission geometry is not feasible except when using very fragile ultra-thin silicon nitride membranes as sample holders. ${ }^{22}$ For the reflection setup, we use either gold coated silica slides or silicon slides, both having a high reflectivity for the used wavelength under reasonable angles of incidence. Another benefit of this approach is that the specimens can simply be pipetted onto the substrate.

The geometry is depicted in Fig. 2. In reflection geometry, the incident light beam having a wavevector $\mathbf{k}_{\text {in }}$ is specularly reflected from the surface of the substrate. An obstacle at the surface scatters the illuminating radiation in all directions causing coherent speckles across the detector. In our experiment, the obstacles are dried cells. They consist mainly of carbon which is highly reflective at XUV wavelengths, resulting in a lot of diffracted light from both the surface and the silhouette of the specimen. According to the Fraunhofer diffraction theory, the diffraction pattern corresponds to the three-dimensional (3D) Fourier transform of the obstacle in the far-field. ${ }^{23}$ In other words, the idea of the current setup is to study the specimen not in the real space but in the Fourier plane. Full details and analytical equations for the scattering geometry are given in Ref. 13 and references therein. In the presented case, a slightly curved

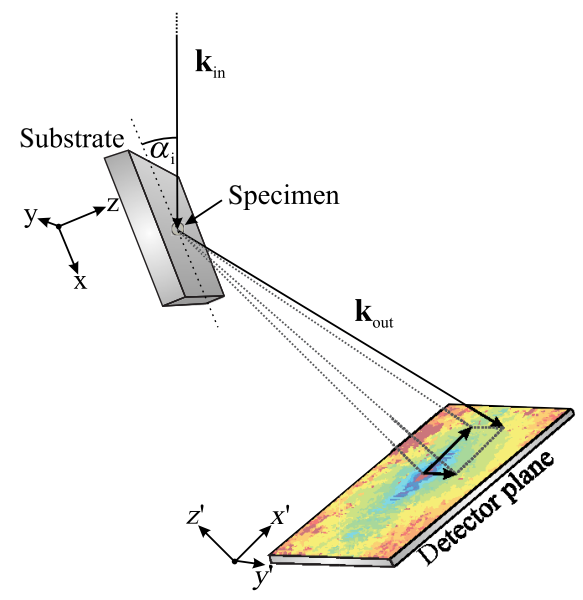

Fig. 2 Detail of the reflection geometry. The incident light beam $\mathbf{k}_{\text {in }}$ is reflected from the substrate and scattered off the specimen. The forward wavevector $\mathbf{k}_{\text {out }}$ hits the detector at a certain pixel. The captured diffraction pattern corresponds to the three-dimensional (3-D) Fourier transform of the object. The angle of incidence $\alpha_{i}$ for the experiments presented here was 22.5 deg to the surface plane. 
wave front is used for illumination. This has the advantage that the inner part of the diffraction pattern, which would otherwise contain the very intense central speckle, corresponds to a low resolution hologram of the specimen. ${ }^{15}$ The less intense central part is beneficial because the full dynamic range of the CCD camera can be effectively used in a single recorded image. In comparison, for pure CDI experiments, high dynamic range images must be stitched together from images taken with different exposure times to counteract saturation of the camera in the center.

\section{Fast Classification Method}

As we will show, reconstructing a real space image is not necessary because the captured diffraction pattern is sufficient as a fingerprint of the biologic specimen. This fingerprint is the far-field of the diffracted light corresponding to the spatial Fourier transform of the object. ${ }^{23}$ The benefit of recording and analyzing data in the Fourier domain is that phase shifts and lateral shifts of the object in respect to the laser beam only change the phase in the Fourier domain, and the phase cancels by recording the intensity ${ }^{24}$ pattern. This makes the measurement insensitive to such shifts and comparison of diffraction patterns is more straightforward.

Since 38-nm light does not penetrate the object, we are only gaining information of the morphology and silhouette of the specimen. Surprisingly, this seems to be sufficient to distinguish between different specimens, as shown in Fig. 3. Please note that the specimens in Fig. 3 are all breast cancer cell line cells, as will be explained in the next section, and exhibit different expression profiles. From these pictures, we can already conclude that our method is sensitive enough to clearly distinguish different cells. Moreover, according to the Fraunhofer diffraction theory, we can expect a more pronounced speckle pattern if the sample shows symmetries or certain morphological features are repeated. Therefore, our approach can also be used for anisotropy analysis, e.g., the SKBR3 cell brings forward a more pronounced anisotropy compared to the MCF7 cells (Fig. 3).

An overview of the proposed approach ${ }^{25}$ for cell classification is depicted in Fig. 4. In a first step, a database containing the diffraction patterns (fingerprints) of different specimens will be established.

For classifying an unknown cell, the coherent XUV light is scattered off the object and the measured diffraction pattern will be compared with the entries in the database. The comparison is based on two-dimensional (2-D) image processing techniques which are widely used nowadays, e.g., for face recognition. This comparison will speed up the evaluation process significantly compared to spatial image reconstruction.

In a first proof-of-principle experiment, we applied a simple 2-D cross-correlation method to numerically compare the different diffraction patterns. ${ }^{26}$ The 2-D cross-correlation $C(i, j)$ of diffraction patterns $A$ and $B$ is given by

$C(i, j)=\sum_{m=0}^{(N-1)} \sum_{n=0}^{(N-1)} A(m, n) \cdot \operatorname{conj}[B(m+i, n+j)]$

with $0 \leq i, j<2 N-1$, where $i$ and $j$ are the vertical and horizontal pixel indices, respectively. From this cross correlation, we compute a correlation factor $V$ with

$V=\max |\nabla[\nabla C(i, j)]|$,

using $\nabla C(i, j)=[\partial C(i, j) / \partial i] \hat{i}+[\partial C(i, j) / \partial j] \hat{j}$. The magnitude of $V$ is a measure of the similarity of the two diffraction patterns.

\section{Sample Preparation}

A great benefit of the presented technique is that no special preparation like staining or cultivation of the cells is necessary. In the following, we will limit ourselves to cancer cells as our targeted specimen. For a first proof-of-principle experiment, we prepared MCF7 and SKBR3 breast cancer cell line cells from a cell culture. These commercially available epithelial-like cell lines were established from the pleural effusions of two Caucasian women (ages 69 and 43 years) with metastatic mammary carcinomas. These specimens further represent a good model to mimic circulating tumor cells in the blood stream of breast cancer patients. ${ }^{27}$ These specific cell line cells have been selected mainly due to their wide availability and not because of any obvious differences in the morphology.

The unstained and unlabeled cells were cultivated in $90 \%$ advanced DMEM and 10\% FBS with 2-mM Glutamine up to $90 \%$ confluency. After trypsinization, cells were washed twice in phosphate-buffered saline (PBS), then they were placed on the substrate using an inverted microscope and a micromanipulator device equipped with a micropipette. As an example, we depict one of the prepared samples containing several MCF7
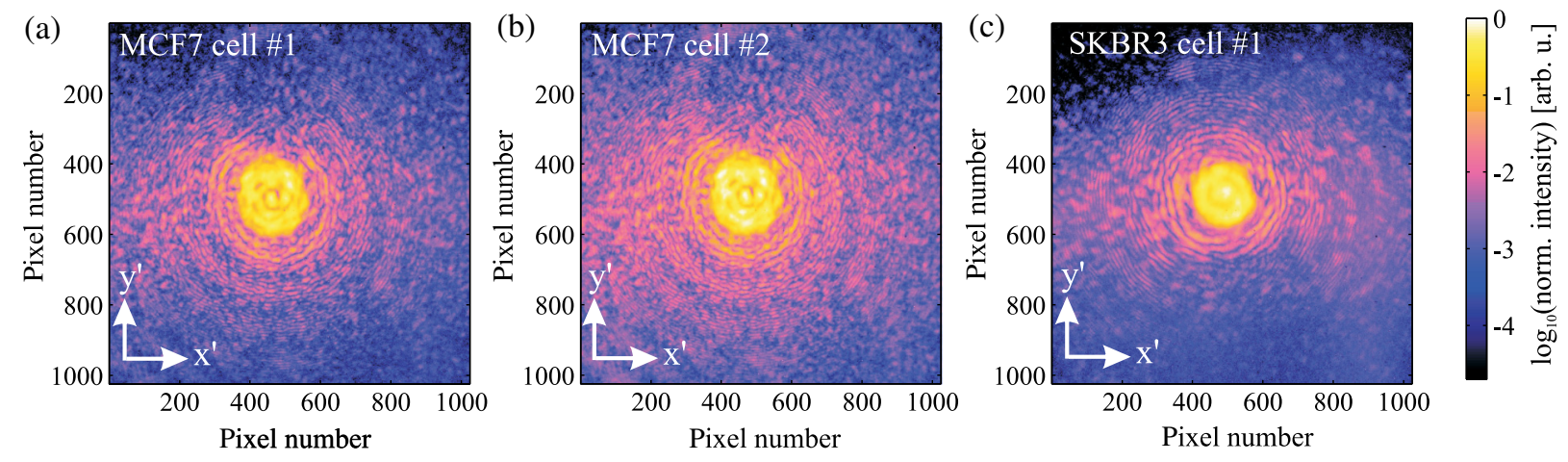

Fig. 3 Three examples for typical two-dimensional (2-D) diffraction patterns obtained from a single cell. The diffraction patterns for the two different MCF7 breast cancer cells [(a) and (b)] are similar, whereas the SKBR3 diffraction pattern (c) looks different. Note the low resolution hologram in the center of each image and the coherent diffraction imaging $(\mathrm{CDI})$ related speckle in the outer parts. 


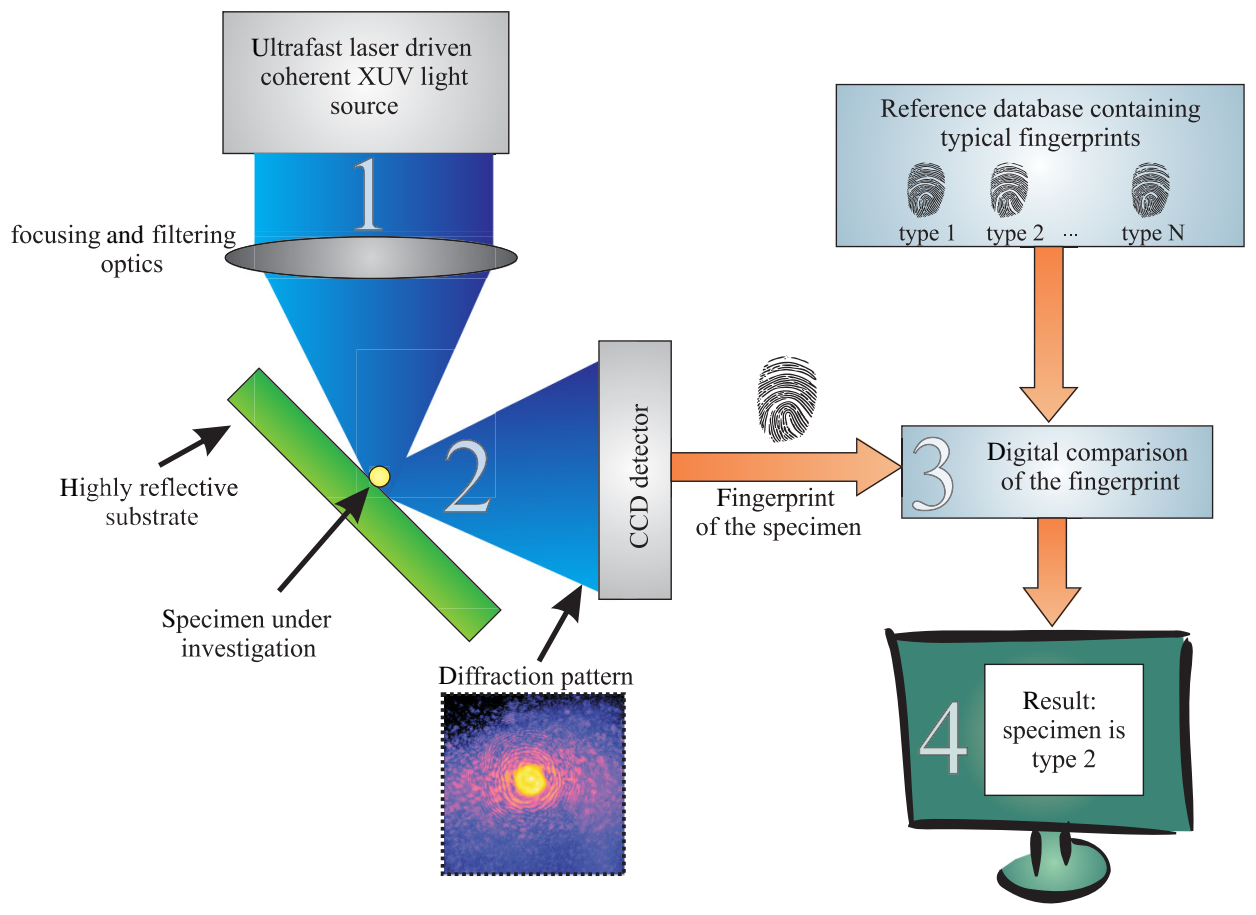

Fig. 4 Sketch of the proposed method for rapid classification of biologic specimens. The coherent XUV source (1) is focused onto the sample (2), which is pipetted onto a reflective substrate, e.g., silicon. A detector captures the scattered light and compares (3) the measured diffraction pattern as a kind of fingerprint with the entries of a database. The result of the classification is then displayed (4).

cells in Fig. 5. After drying the cell, the salt forms small crystalline structures around the cells. As long as they are well separated from the cell, they do not impair the measurements, because the focal spot of the XUV beam is small enough to only illuminate the cell and the diffraction pattern only contains information of the morphology of the cell.

\section{Measurements and Results}

The coherent XUV light was scattered off each cell and the 2-D fingerprints were subsequently captured using an exposure time

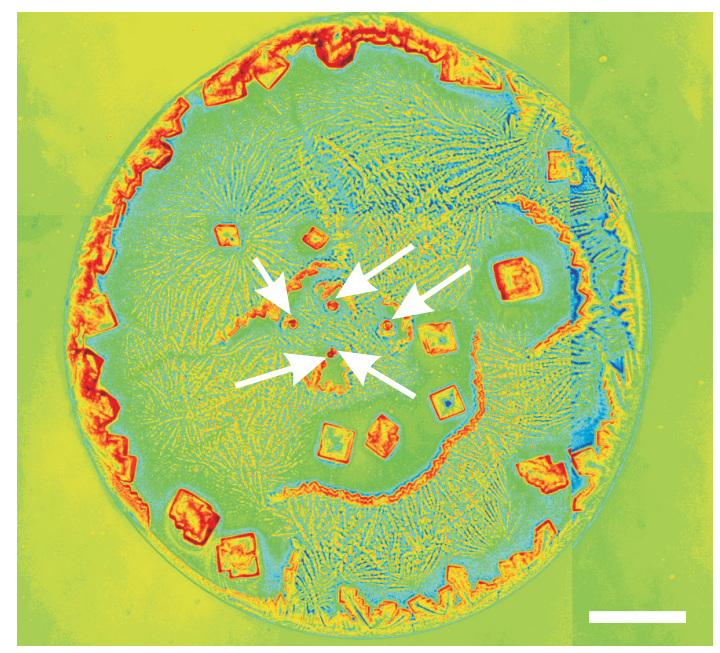

Fig. 5 Light microscope image after the phosphate-buffered saline (PBS) buffer containing several cancer cells dried out on a gold coated substrate. Adjacent to the MCF7 cells (marked by arrows) salt crystals are clearly visible on the substrate. The scale bar corresponds to $200 \mu \mathrm{m}$. of $1500 \mathrm{~s}$. The recorded pictures were then compared with the described cross-correlation technique. The magnitude of the correlation factor $V$ enables us to judge whether the cells are from the same cell line or not. The results of the comparison for four different cells are summarized in Table 1. Setting the threshold properly $(V>3.5$ was experimentally determined

Table 1 Correlation factor $V$ of four different diffraction patterns of MCF7/SKBR3 breast cancer cell line cells (upper table). Setting the threshold at $V=3.5$ results in classification of the cell expression, see lower table. Cells are classified as identical " $=$ " or nonidentical " $\neq$."

\begin{tabular}{lcccc}
\hline \multicolumn{5}{c}{ Cell classification } \\
& MCF7 & MCF7 & MCF7 & SKBR3 \\
& cell \#1 & cell \#2 & cell \#3 & cell \#1 \\
\hline & & $V$ & & \\
MCF7 cell \#1 & 13.03 & 9.48 & 3.75 & 1.99 \\
MCF7 cell \#2 & 9.48 & 15.52 & 4.49 & 2.16 \\
MCF7 cell \#3 & 3.75 & 4.49 & 13.67 & 3.13 \\
SKBR3 cell \#1 & 1.99 & 2.16 & 3.13 & 7.95 \\
& & Match? & & \\
MCF7 cell \#1 & $=$ & $=$ & $=$ & $\neq$ \\
MCF7 cell \#2 & $=$ & $=$ & $=$ & $\neq$ \\
MCF7 cell \#3 & $=$ & $=$ & $\neq$ & $\neq$ \\
SKBR3 cell \#1 & $\neq$ & $\neq$ & & $\neq$
\end{tabular}


(a)

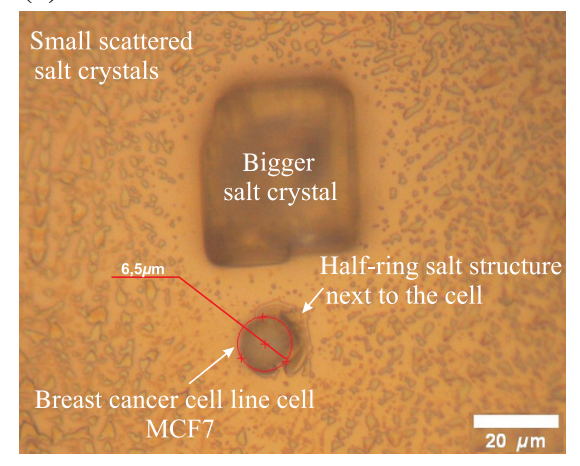

(b)

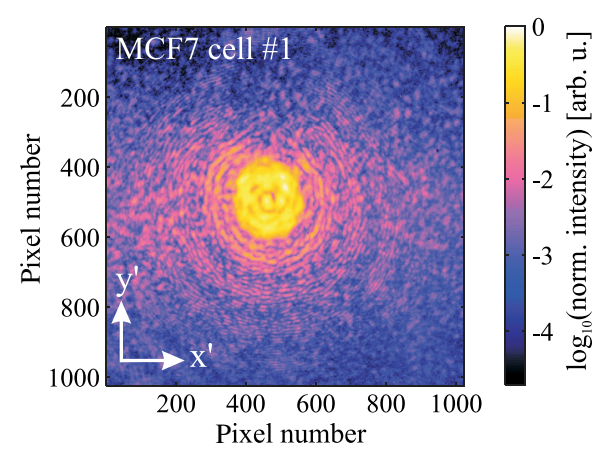

(c)

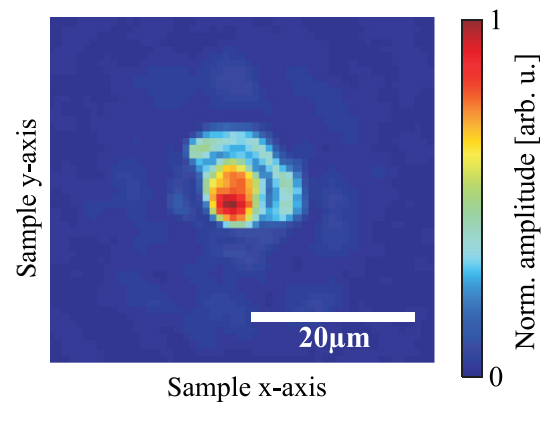

Fig. 6 (a) Light microscope image (50x magnification) of the breast cancer cell line cell (MCF7) on a gold substrate, surrounded by scattered salt crystals. (b) Measured diffraction pattern upon reflecting the coherent 38-nm beam off the cell. (c) Reconstructed real space image featuring the MCF7 cell and the half-ring of salt.

for this specific case) allows us to finally distinguish between the different cell expressions from the diffraction pattern solely without any a priori knowledge about the type of cell. It should be mentioned that with more sophisticated image comparison techniques, e.g., feature-based image comparison, it will also be possible to distinguish cells with a more similar morphology. Unfortunately, to our best knowledge, there exists no quantitative comparison of the MCF7 and SKBR3 morphology obtained with other methods such that the measured effect could be correlated to an actual difference in morphology for the used cell expressions.

Despite a spatial reconstruction not being necessary to classify the cell expressions, it may be beneficial to compute a real space image of the specimen once an interesting specimen has been found by the classification method. Therefore, we use wellestablished iterative phase retrieval algorithms to reconstruct the phase of the diffraction pattern. ${ }^{18}$ Once the phases are reconstructed, we can compute the real space image by a simple Fourier transform. A detailed description of the reconstruction algorithm used can be found in Ref. 13 and references therein.

In Fig. 6, we present an example for a reconstruction. On the image [Fig. 6(a)] taken with a conventional light microscope, one can clearly see the salt crystals from the PBS buffer around the MCF7 cell. In our experiment, the XUV beam has a diameter sufficient to simultaneously illuminate the cell itself and the small salt crystal next to it. Hence, both of them form the diffraction pattern shown in Fig. 6(b). Subsequently, the reconstruction [Fig. 6(c)] clearly shows the reconstructed cell and the small salt crystal in the shape of a half-ring. The resolution of the reconstructed image is slightly below one micron and is mainly limited by the relatively low numerical aperture of the imaging system in the current experiment.

Other reconstructed images from MCF7 cells are similar but most of them are surrounded by crystals with different shapes. Despite the distortions introduced by these surrounding crystals, it is still possible to classify the cells with the recorded diffraction pattern. With an increased spatial resolution, it should be possible to trace down the actual morphology of the cells and relate this to the features obtained in the diffraction pattern in the near future.

A major concern when dealing with imaging of biologic specimens in the XUV and x-ray regimes is radiation damage. The presented experiment was repeated several times at different days on the same individual cells and resulted in comparable diffraction patterns ruling out radiation damage. For the $1500 \mathrm{~s}$ long exposure, we estimated the dose to $\sim 10^{9} \mathrm{~Gy}$, which is below the damage threshold. ${ }^{28}$ Since only the number of scattered photons off the sample is important for the formation of the diffraction pattern, increasing the flux allows shortening the exposure time, ensuring a dose below the damage threshold.

\section{Conclusion and Discussion}

We are confident that the demonstrated approach, accompanied by the simple classification method, will pave the way toward a fast cell classification instrument fitting into every hospital. Further, this method could be easily extended to classify bacteria and viruses since the short wavelength supports a spatial resolution comparable to their size. Currently, the major limitation for the routine application of this technique is the long integration time, which could be drastically reduced by increasing the photon flux from the HHG source through using novel high repetition rate sources. ${ }^{29}$ An even better discrimination of cells will be possible if the cells can be classified in their natural environment. This will be possible by using more powerful lasers, allowing the generation of coherent XUV radiation in the water window (4.4 to $2.2 \mathrm{~nm}){ }^{12}$ where water is only weakly absorbing the XUV radiation. In the water window, diffraction patterns can be captured in combination with a flow cytometer, which would allow the counting and classification of circulating tumor cells at a high throughput.

\section{Acknowledgments}

Support from the European Regional Development Fund (EFRE) and TMBWK under Grant No. B 715-08008 is acknowledged. S. F. acknowledges support from Siemens Healthcare.

\section{References}

1. H. Koyi, G. Hillerdal, and E. Branden, "Patient's and doctors' delays in the diagnosis of chest tumors," Lung Cancer 35(1), 53-57 (2002).

2. R. Petry, M. Schmitt, and J. Popp, "Raman spectroscopy-a prospective tool in the life sciences," Chem. Phys. Chem. 4(1), 14-30 (2003).

3. J. Miao et al., "Extending the methodology of X-ray crystallography to allow imaging of micrometre-sized non-crystalline specimens," Nature 400(6742), 342-344 (1999).

4. H. N. Chapman and K. A. Nugent, "Coherent lensless X-ray imaging," Nat. Photonics 4(12), 833-839 (2010). 
5. S. Boutet et al., "High-resolution protein structure determination by serial femtosecond crystallography," Science 337(6092), 362-364 (2012).

6. L. Redecke et al., "Natively inhibited trypanosoma brucei Cathepsin B structure determined by using an X-ray laser," Science 339(6116), 227-230 (2013).

7. B. Abbey et al., "Lensless imaging using broadband X-ray sources," Nat. Photonics 5(7), 420-424 (2011).

8. M. M. Seibert et al., "Femtosecond diffractive imaging of biological cells," J. Phys. B At. Mol. Opt. 43(19), 194015 (2010).

9. A. P. Mancuso et al., "Coherent imaging of biological samples with femtosecond pulses at the free-electron laser FLASH," New J. Phys. 12, 035003 (2010).

10. T. Brabec and F. Krausz, "Intense few-cycle laser fields: frontiers of nonlinear optics," Rev. Mod. Phys. 72(2), 545-591 (2000).

11. R. A. Bartels et al., "Generation of spatially coherent light at extreme ultraviolet wavelengths," Science 297(5580), 376-378 (2002).

12. J. Seres et al., "High-harmonic generation and parametric amplification in the soft X-rays from extended electron trajectories," Sci. Rep. 4(4234), 1-7 (2014).

13. M. Zürch, C. Kern, and C. Spielmann, "XUV coherent diffraction imaging in reflection geometry with low numerical aperture," Opt. Express 21(18), 21131-21147 (2013).

14. M. Zürch and C. Spielmann, "Verfahren und Vorrichtung zur Erzeugung einer schmalbandigen, kurzwelligen, kohärenten Laserstrahlung, insbesondere für XUV-Mikroskopie," Patent pending, Germany DE102012022961.5 (2012)

15. G. J. Williams et al., "Fresnel coherent diffractive imaging," Phys. Rev. Lett. 97(2), 025506 (2006).

16. L. W. Whitehead et al., "Fresnel diffractive imaging: experimental study of coherence and curvature," Phys. Rev. B 77(10), 104112 (2008).

17. A. V. Martin et al., "Femtosecond dark-field imaging with an X-ray free electron laser," Opt. Express 20(12), 13501-13512 (2012).

18. J. R. Fienup, "Phase retrieval algorithms: a personal tour," Appl. Opt. 52(1), 45-56 (2013).

19. S. Marchesini et al., "X-ray image reconstruction from a diffraction pattern alone," Phys. Rev. B 68(14), 140101 (2003).

20. D. F. Gardner et al., "High numerical aperture reflection mode coherent diffraction microscopy using off-axis apertured illumination," Opt. Express 20(17), 19050-19059 (2012).

21. S. Roy et al., "Lensless X-ray imaging in reflection geometry," Nat. Photonics 5(4), 243-245 (2011).

22. B. Zhang et al., "Full field tabletop EUV coherent diffractive imaging in a transmission geometry," Opt. Express 21(19), 21970-21980 (2013).

23. H. N. Chapman et al., "High-resolution ab initio three-dimensional x-ray diffraction microscopy," J. Opt. Soc. Am. A 23(5), 1179-1200 (2006).

24. R. H. T. Bates, "Fourier phase problems are uniquely solvable in more than one dimension. I: underlying theory," Optik 61, 247-262 (1982).
25. M. Zürch and C. Spielmann, "Verfahren zur Auswertung von durch schmalbandige, kurzwellige, kohärente Laserstrahlung erzeugten Streubildern von Objekten, insbesondere zur Verwendung in der XUVMikroskopie," Patent pending, Germany DE 102012022961.5 (2012).

26. D. M. Tsai and C. T. Lin, "Fast normalized cross correlation for defect detection," Pattern Recogn. Lett. 24(15), 2625-2631 (2003).

27. C. Arya et al., "Capturing rare cells from blood using a packed bed of custom-synthesized chitosan microparticles," J. Mater. Chem. 1(34), 4313-4319 (2013).

28. M. R. Howells et al., "An assessment of the resolution limitation due to radiation-damage in x-ray diffraction microscopy," J. Electron. Spectrosc. Relat. Phenom. 170(1-3), 4-12 (2009).

29. J. Rothhardt et al., " $53 \mathrm{~W}$ average power few-cycle fiber laser system generating soft $\mathrm{x}$ rays up to the water window," Opt. Lett., 39(17), 52245227 (2014).

Michael Zürch received his diploma degree in 2010 on optical induced damage in nanostructures at the Friedrich-Schiller-University in Jena. He completed his PhD degree in 2014 at the Institute of Optics and Quantum Electronics at FSU Jena on the generation of coherent laser-like extreme ultraviolet (XUV) light and its application to high-resolution imaging.

Stefan Foertsch received his diploma degree in chemistry at the Friedrich-Alexander-University Erlangen (Prof. Dr. Dirk Guldi) in cooperation with Siemens Healthcare, in 2010. The topic was "Development of a new electrochemical sensor for detection of Helicobacter pylori." Currently, he is finishing his PhD degree "Development of new in vivo and in vitro sensors for the Gl tract" at the Department of Physical Chemistry at Friedrich-AlexanderUniversity Erlangen in cooperation with Siemens Healthcare.

Rainer Kuth studied physics and chemistry at the University of Düsseldorf and has been working in industry since 1986 in various roles. He has published over 500 innovations for medical applications with over 200 coauthors. Currently, he is responsible for projects developing disruptive new business models in medical technology.

Christian Spielmann received his PhD degree in 1992 at the TU Vienna. After postdoc stays at the University of California in San Diego and the TU Vienna, he habilitated in 1999 in Vienna. In 2001, he went to the University of Würzburg as a professor. Since 2008, he is chair of the Quantum Electronics Department at the University of Jena. Besides his research activities, he is the director of the Abbe Center of Photonics and speaker of the Graduate School of the Helmholtz Institute Jena.

Biographies of the other authors are not available. 